\title{
Family Characteristics and Quality of Life of Substance Abusing Clients at a Nigerian Rehabilitation Center
}

\author{
Aishatu Yusha'u Armiya'u ${ }^{1, ~ *, ~ L u b u o l a ~ I s s a ~ B a m i d e l e ~}{ }^{1}$, Zuwaira Hassan ${ }^{2}$, Francis John Davou ${ }^{3}$ \\ ${ }^{1}$ Department of Psychiatry, Jos University Teaching Hospital, Jos, Nigeria \\ ${ }^{2}$ Department of Community Medicine, University of Jos/Jos University Teaching Hospital, Jos, Nigeria \\ ${ }^{3}$ Department of Psychiatry, University of Abuja, Abuja, Nigeria
}

Email address:

aarmiyau@gmail.com (A. Y. Armiya’u)

${ }^{*}$ Corresponding author

\section{To cite this article:}

Aishatu Yusha'u Armiya'u, Lubuola Issa Bamidele, Zuwaira Hassan, Francis John Davou. Family Characteristics and Quality of Life of Substance Abusing Clients at a Nigerian Rehabilitation Center. Psychology and Behavioral Sciences. Vol. 8, No. 1, 2019, pp. 1-8. doi: 10.11648/j.pbs.20190801.11

Received: October 30, 2018; Accepted: November 12, 2018; Published: January 19, 2019

\begin{abstract}
The study was aimed at assessing the family characteristics and the relationship between family characteristics and objective as well as subjective quality of life among participants using single substance versus multiple substance users. The study is part of a comprehensive cross sectional descriptive study assessing 190 participants who consented to be studied. Each participant completed a family characteristic and subjective quality of life questionnaire along-side a World Health Organization Quality of Life-BREF questionnaire. All participants were interviewed with the Composite International Diagnostic Interview instrument to make a diagnosis of substance use disorder using ICD-10 diagnostic criteria. The data was analyzed using the Statistical Package for Social Sciences Version 20.0. The study found multiple substance users whose fathers had primary school education $(p=0.006)$ and had died $(p=0.027)$ had lower quality of life in their social relationship domain on World Health Organization Quality of Life. No statistical significant relationship was found between subjective quality of life and family characteristics of single versus multiple substance users. In conclusion only social relationship domain on World Health Organization Quality of Life was found to have statistically significant relationship in the study. All other domains on World Health Organization Quality of Life were not significantly associated with any family characteristic.
\end{abstract}

Keywords: Nigeria, Quality of Life, Rehabilitation Center, Family Characteristics, Substance Abuse

\section{Introduction}

Quality of Life (QOL) is increasingly recognized as cardinal to the generic construct of recovery in Substance abuse services [1].

Assessing QOL in substance users can complement more objective symptom measure, identify special service needs and document changes in functioning that are associated with substance use patterns.

The World Health Organization (WHO) defined QOL as an individual's perception of their position in life in the context of the culture and value systems in which they live and in relation to their goals, expectations, standards and concerns [2]. The domains typically included in the definitions of behavioural health recovery are physical and mental health, social functioning and living environment which include; safety, comfort, convenience of living environment and access to available resources. These domains are cited by individuals in recovery as key priorities and consistent with expert guiding criteria for Substance Use Disorders treatment evaluation [3].

Recovery from addiction integrally incorporated improved health wellness and quality of life, as defined by the Substance Abuse and Mental Health Services Administration [4].

It is worthy of note that substance use may negatively impact on QOL through increased symptoms and greater 
distress, for instance, by exacerbating symptoms and or interfering with treatment [5].

More importantly, the influence of family characteristics on quality of life will be a somewhat interesting modifiable factor in predicting QOL among substance users. This is because family characteristics may be easily ignored and left out of consideration in QOL assessment in substance abusers. In support of this, studies conducted among substance users largely assessed family characteristics as predictors of substance use with little or no attention paid to the potential impact of the characteristics such as type of family, parents living status, marital status of parents, substance use in the family and highest level of education of parents among others on QOL of substance abusers [1, 5-9] - a major determinant of recovery.

In the same vein, a study closely related to this study was conducted in Sweden to assess predictors of QOL among substance users [6]. However, the study did not include family characteristics of the participants among the variables considered in the test of association with the quality of life.

Moreover, there has been increased interest in the QOL as an outcome measure, especially in addiction treatment, yet influence of family characteristics on QOL remains an under investigated area especially in the low and middle income resource settings including Nigeria. The unanswered question remains whether family characteristics will significantly impact on the QOL of substance users.

In the light of the above, this study examined the potential influence of family characteristics on the QOL of substance users at a treatment facility in the North- Central Nigeria so as to bridge the gap from previous studies and lay foundation for others in the area of Addiction Psychiatry.

\section{Method}

\subsection{Study Design and Location}

A cross sectional, descriptive study was conducted in two stages among 190 substance abusing participants at the Substance Abuse Treatment and Rehabilitation Center Jos, Plateau state Nigeria.

\subsection{Data Collection}

In the first stage, every participant was given a questionnaire booklet containing a subjective quality of life questionnaire and a questionnaire for family characteristics of participants alongside the WHOQOL-BREF questionnaires, an instrument that has been validated among various populations and used in Nigeria [2]. The second stage consists of the application of the substance use disorder module of Composite International Diagnostic Interview (CIDI). The instrument has been validated by researchers in different countries including Nigeria [10-11].

\subsection{Instruments}

\subsubsection{Family Characteristic Questionnaire}

The family characteristic questionnaire was designed by the authors to elicit information from the participants on variables such as type of family, parents living status, marital status of parents, substance use in the family and highest level of education of parents among others.

\subsubsection{Subjective Quality of Life Questionnaire}

The subjective quality of life questionnaire was used to elicit information about the participants' neighbourhood, access to social amenities among others which was adopted from Adeponle.

\subsubsection{World Health Organization Quality of Life - BREF (WHOQOL-BREF)}

This instrument was used to collect data on the objective quality of life of all consenting respondents. It is a short version of the self-report questionnaire (WHOQOL - 100) designed by the WHO to measure individual's perception of their position in life in the context of the culture and value systems in which they live and in relation to their goals, expectations, standards and concerns [2].

\subsubsection{Composite International Diagnostic Interview (CIDI)}

The Substance Section was administered to all the clients who consented to participate in the study and fulfill the inclusion criteria. CIDI is a comprehensive, fully standardized interview that can be used to assess mental disorder and provide diagnosis according to the definition and criteria of the tenth revision of the International Classification of Diseases (ICD10) and the fourth edition of the Diagnostic and Statistical Manual of mental disorder (DSM IV) American Psychiatric Association [12-13].

\subsection{Analysis}

Data was analyzed using the Statistical Package for Social Sciences (SPSS) version 20.0. The frequency distribution of these variables, means and standard deviation were calculated and a probability of 5\% was regarded as statistically significant.

\section{Results}

\subsection{Characteristics of Participants' Family}

The marital status of participants' parents showed that 146 (77\%) were married. Almost half 90 (47\%) of the participants' fathers had some form of tertiary education. Most of the participants' fathers were employed 142 (75\%). One hundred and six (56\%) of participants mothers' were employed (Table 1).

One hundred and forty six (77\%) of the participants were from monogamous family, while 44 (23\%) from polygamous families, (Table 1). Most of the participants $133(70 \%)$ had their fathers alive, while $57(30 \%)$ had lost their fathers, (Table 1). For the mothers, 88 (46\%) had some form of 
tertiary education, $44(23 \%)$ secondary education, $18(10 \%)$ primary education, while $40(21 \%)$ had no formal education, (Table 1). Only a few of the participants lost their mothers 18 (10\%), the remaining 172 (90\%) had their mothers alive, (Table 1). Most of the family members 102 (54\%) did not abuse alcohol or psychoactive drugs, while 88 (46\%) abused psychoactive substances, (Figure 1). Out of the 86 family members who abused substances 54 (63\%) abused alcohol, $12(14 \%)$ tobacco, $18(21 \%)$ marijuana and $1(1 \%)$ each abused opiate and sedatives respectively, (Figure 2).

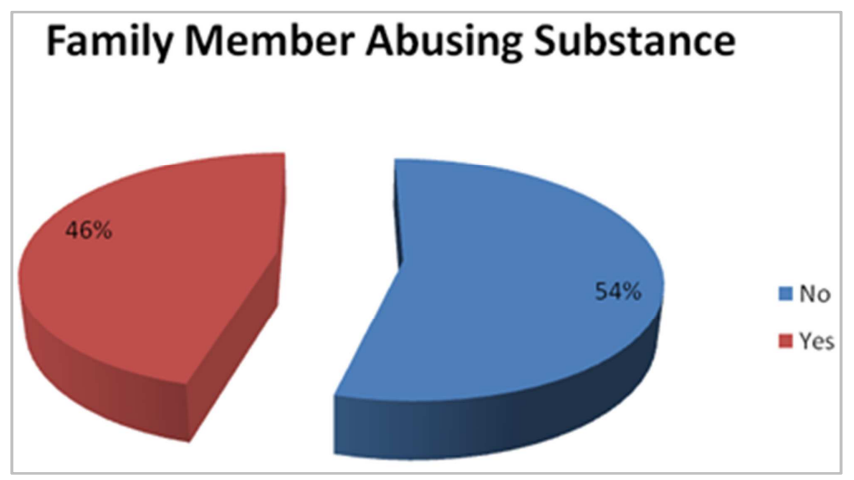

Figure 1. Pie chart showing family members of participants abusing substances.

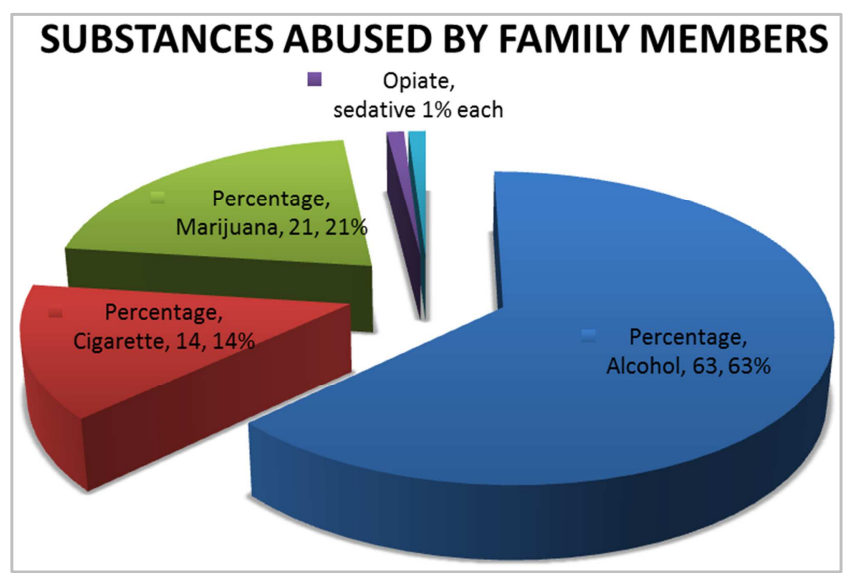

Table 1. Characteristics of participants' family.

\begin{tabular}{lll}
\hline Variables & Frequency 190 & Percentage 100\% \\
\hline Type of family & & \\
Monogamous & 146 & 77 \\
$\begin{array}{l}\text { Polygamous } \\
\text { Living status of fathers }\end{array}$ & 44 & 23 \\
Alive & 133 & 70 \\
Not alive & 57 & 30 \\
Living status of mothers & & \\
Alive & 172 & 90 \\
Not alive & 18 & 10 \\
Marital status & & \\
Married & 146 & 77 \\
Divorced & 6 & 3 \\
Separated & 38 & 20 \\
Fathers'educational level & & \\
Non formal & 32 & 18 \\
Primary & 19 & 10 \\
Secondary & 49 & 25 \\
Tertiary & 90 & 47 \\
Mothers'educational level & & \\
Non formal & 40 & 21 \\
Primary & 18 & 10 \\
Secondary & 44 & 23 \\
Tertiary & 88 & 46 \\
Mothers'employment status & & \\
Employed & 106 & 56 \\
Unemployed & 73 & 38 \\
Retired & 11 & 6 \\
\hline & &
\end{tabular}

\subsection{Subjective Quality of Life of Participants}

More than half of the participants $123(65 \%)$ lived in medium density neighborhood with $19(10 \%)$ living in low density neighborhood. Concerning psychological states, most of the participants experienced a depressed mood sometimes 132(74\%), 37(15\%) not at all, while 21(11\%) experienced depressed mood frequently. One hundred and forty two (75\%) experienced anxiety sometimes, $35(18 \%)$ did not, while $13(7 \%)$ had anxiety symptoms frequently. Less than half of the participants $85(45 \%)$ had fair concentration, 30(16\%) concentrated poorly, while 75(39\%) concentrated well (Table 2 ).

Figure 2. Substances abused by family members of subjects.

Table 2. Subjective quality of life of participants.

\begin{tabular}{|c|c|c|c|}
\hline & Participants indicators & $N=190$ Frequency & Percentage (100\%) \\
\hline & Low density & 19 & 10 \\
\hline \multirow{3}{*}{ Type of neighbourhood } & Medium density & 123 & 65 \\
\hline & High density & 48 & 25 \\
\hline & Electricity & & \\
\hline \multirow[t]{10}{*}{ Housing conditions } & Yes & 165 & 87 \\
\hline & No & 25 & 13 \\
\hline & Pipe borne water & & \\
\hline & No & 84 & 44 \\
\hline & Toilet & & \\
\hline & Yes & 165 & 86 \\
\hline & No & 25 & 14 \\
\hline & Telephone & & \\
\hline & Yes & 119 & 63 \\
\hline & No & 71 & 37 \\
\hline
\end{tabular}




\begin{tabular}{|c|c|c|c|}
\hline & Participants indicators & $\mathrm{N}=190$ Frequency & Percentage $(100 \%)$ \\
\hline \multirow{6}{*}{ Relationship with opposite gender } & Not at all & 10 & 5 \\
\hline & Very difficult & 32 & 17 \\
\hline & Easily & 112 & 59 \\
\hline & Not at all & 11 & 6 \\
\hline & Very difficult & 67 & 35 \\
\hline & Finance & & \\
\hline \multirow{12}{*}{ Social support } & Yes & 133 & 70 \\
\hline & No & 57 & 30 \\
\hline & Food & & \\
\hline & Yes & 127 & 67 \\
\hline & No & 63 & 33 \\
\hline & Encouragement/advice & & \\
\hline & Yes & 159 & 84 \\
\hline & No & 31 & 16 \\
\hline & Visit & & \\
\hline & Yes & 129 & 68 \\
\hline & No & 61 & 32 \\
\hline & Outdoor games & & \\
\hline \multirow[t]{12}{*}{ Opportunity/strength } & Yes & 130 & 68 \\
\hline & No & 60 & 32 \\
\hline & Indoor games & & \\
\hline & Yes & 94 & 49 \\
\hline & No & 96 & 51 \\
\hline & Travel/vocation & & \\
\hline & Yes & 92 & 48 \\
\hline & No & 98 & 52 \\
\hline & Frequently & 21 & 11 \\
\hline & Not at all & 37 & 15 \\
\hline & Sometimes & 132 & 74 \\
\hline & Frequently & 13 & 7 \\
\hline \multirow[t]{3}{*}{ Anxiety } & Not at all & 35 & 18 \\
\hline & Sometimes & 142 & 75 \\
\hline & Fair & 85 & 45 \\
\hline \multirow[t]{2}{*}{ Concentration } & Poor & 30 & 16 \\
\hline & Well & 75 & 39 \\
\hline
\end{tabular}

\subsection{Comparison Between Family Characteristics and Mean Scores of the Social Relationship Domain of Whoqol-Bref in Multiple and Single Substance Users}

The study found statistical significant relation in only the social relationship domain of the WHOQOL-BREF for multiple and single substance abusers. A relationship was found between multiple substance abusing participants who lost their fathers $(p=0.027)$, and those whose fathers had only primary school education $(p=0.006)$ with the social relationship domain of the WHOQOL-BREF scale. It also found lower quality of life in the social relationship domain of subjects abusing single substance but having no family member abusing substance (Table 3).

Table 3. Comparison between family characteristics and mean scores of the social relationship domain of whoqol-bref in multiple and single substance users.

\begin{tabular}{|c|c|c|c|c|c|}
\hline \multirow{2}{*}{ Variables } & \multirow{2}{*}{$\begin{array}{l}\text { Multiple users } \\
\text { Mean } \pm \text { SD }\end{array}$} & \multirow{2}{*}{$\begin{array}{l}\text { Single users } \\
\text { Mean } \pm \text { SD }\end{array}$} & \multirow{2}{*}{$\mathbf{F}$} & \multirow{2}{*}{ df } & \multirow{2}{*}{$\mathbf{P}$} \\
\hline & & & & & \\
\hline \multicolumn{6}{|l|}{ Family type } \\
\hline Monogamous & $14.26 \pm 3.51$ & $14.12 \pm 3.15$ & 0.259 & 1,186 & 0.611 \\
\hline Polygamous & $14.35 \pm 3.64$ & $13.86 \pm 3.60$ & & & \\
\hline \multicolumn{6}{|c|}{ Marital status of parents } \\
\hline Married & $14.19 \pm 3.65$ & $13.87 \pm 3.24$ & 0.015 & 4,184 & 0.985 \\
\hline Separated & $14.93 \pm 2.87$ & $14.71 \pm 3.30$ & & & \\
\hline \multicolumn{6}{|l|}{ Father } \\
\hline Alive & $14.90 \pm 3.31$ & $14.02 \pm 3.21$ & 4.966 & 1,186 & $0.027 *$ \\
\hline Not alive & $12.52 \pm 3.61$ & $14.12 \pm 3.36$ & & & \\
\hline \multicolumn{6}{|c|}{ Educational level of father } \\
\hline Non formal & $12.09 \pm 3.36$ & $14.12 \pm 2.31$ & 4.237 & 2,182 & $0.006^{*}$ \\
\hline Primary & $12.00 \pm 2.39$ & $15.08 \pm 3.59$ & & & \\
\hline Secondary & $14.46 \pm 3.07$ & $14.07 \pm 3.32$ & & & \\
\hline \multicolumn{6}{|c|}{ Employment status of father } \\
\hline Unemployed & $14.11 \pm 3.59$ & $13.91 \pm 3.34$ & 0.078 & 3,184 & 0.925 \\
\hline
\end{tabular}




\begin{tabular}{|c|c|c|c|c|c|}
\hline \multirow{2}{*}{ Variables } & \multirow{2}{*}{$\begin{array}{l}\text { Multiple users } \\
\text { Mean } \pm \text { SD }\end{array}$} & \multirow{2}{*}{$\begin{array}{l}\text { Single users } \\
\text { Mean } \pm \text { SD }\end{array}$} & \multirow{2}{*}{$\mathbf{F}$} & \multirow{2}{*}{ df } & \multirow{2}{*}{$\mathbf{P}$} \\
\hline & & & & & \\
\hline Employed & $14.81 \pm 3.74$ & $14.25 \pm 3.14$ & & & \\
\hline Retired & $14.67 \pm 3.21$ & $14.89 \pm 2.72$ & & & \\
\hline \multicolumn{6}{|l|}{ Mother } \\
\hline Alive & $14.28 \pm 3.57$ & $14.18 \pm 3.16$ & 0.877 & 3,186 & 0.350 \\
\hline Not alive & $14.33 \pm 3.32$ & $12.67 \pm 3.99$ & & & \\
\hline \multicolumn{6}{|c|}{ Educational level of mother } \\
\hline Primary & $14.00 \pm 4.71$ & $13.83 \pm 3.86$ & & & \\
\hline Secondary & $14.92 \pm 3.74$ & $14.19 \pm 3.79$ & & & \\
\hline Tertiary & $14.79 \pm 3.26$ & $14.21 \pm 3.17$ & & & \\
\hline \multicolumn{6}{|c|}{ Employment status of mother } \\
\hline Unemployed & $14.50 \pm 3.49$ & $13.87 \pm 3.29$ & 0.425 & 2.184 & 0.654 \\
\hline Employed & $13.90 \pm 3.66$ & $14.24 \pm 2.99$ & & & \\
\hline Retired & $14.67 \pm 3.53$ & $14.50 \pm 4.53$ & & & \\
\hline Yes & $14.00 \pm 3.55$ & $14.73 \pm 3.20$ & 4.347 & 1,186 & $0.038^{*}$ \\
\hline No & $14.77 \pm 3.48$ & $13.38 \pm 3.18$ & & & \\
\hline
\end{tabular}

*significant value;

$\mathrm{df}=$ degree of freedom;

$\mathrm{F}=$ 2-way Anova;

Statistically significant $\mathrm{p}<0.05$

\section{Discussion}

The research looked at the marital status of parents and found over 7 in 10 of the parents were married, smaller fractions were separated. This is in contrast to another study which found (38\%) of the parents as divorced [14]. Children raised in intact married families are more likely to be physically and emotionally healthier and less likely to use drugs or alcohol and to commit delinquent behaviours; this study found a contrary view. Most subjects in this study were found to be from married families with both parents having tertiary education and employed. This might suggest that subjects in the study had more money to spend and have a lot of peer influence as their parents are mostly at work. Importance of peer influence and high income has previously been reported as factors associated with drug use among adolescents [15]. Parental conflicts, divorce or separation renders the youngster vulnerable to substance abuse. This is because children experience diminished psychological wellbeing, greater unhappiness, less satisfaction with their life, weaker sense of personal control which could eventually lead to anxiety, depression and substance use in these youngsters [16].

Death of a parent or both parents was also studied in the research and it was found that more than half of the fathers were alive with a third of them dead; almost all the mothers in the study were alive. This is similar to a study in Ghana which found that a quarter $(25 \%)$ of the fathers were dead with almost half (45\%) losing both parents. Parents play the greatest role in development of the child in the home and the family [17]. This role involves primarily a source of emotional warmth, comfort and socialization for the child. Loss of parent or both parents through death or long separation usually has a negative impact on the behavior of the growing child especially where there is no appropriate substitute. This negative effect manifests itself more with the loss of father $(25.3 \%)$ than mothers $(10.3 \%)$. This may be due to lack of paternal authority or role model in such unfortunate situation [14].

The presence of substance use in the family seems to be important because almost half of the subjects had a family member or more using substances. The finding is similar to a study conducted in Pakistan by Lashkaripour et al [18] though their figure was higher $(63 \%)$, but in contrast to other studies that found lower rates (24.8\%) and (26\%) respectively $[14,19]$. According to Newcomb and Bentler, youths are more apt to use substances when parents or siblings are heavy users of alcohol or recreationally use illegal drugs [20].

More than half of the subjects lived in medium density neighbourhood with few living in low density neighbourhood. Middle and low density neighbourhood attachment means that community residents have little connection to others in the neighbourhood, parents don't talk to each other, and few community norms exist regarding such issues as curfew and drinking [21]. This community disorganization can occur in middle and upper-middle class neighbourhood as well as lower socioeconomic settings [21].

The research found that a small percentage (11\%) of the subjects experienced depressed mood frequently which is similar to a study which found that depressive symptoms were present in (18\%) of their subjects [22]. Multiple substance abuse has been noted as one of the risk factors for psychiatric morbidity in substance abuse [23]. This may be due to the pharmacological toxicities of the substances, acute intoxication, or as a consequence of withdrawal from chronic use [23]. Another study examined the co-morbidity of dependence on single and multiple substances with emotional disorders and found that the risk for psychiatric morbidity was greater among individuals who were uniquely abusing cigarette, alcohol or cannabis [22].

A greater number of subjects using single substance reside 
in high density neighbourhood compared to those abusing multiple substances but not statistically significant. The majority of this subjects use alcohol, neighbourhood may influence youth alcohol use through indirect pathways. Youths who live in disadvantaged neighbourhoods may have more access to alcohol which has been found to increase alcohol use [24]. Communities marked by low quality housing and high level of disorganization - vandalism, broken windows, and unoccupied dwelling- experience higher levels of drug abuse [21]. Factors that contribute to a person's propensity to abuse drug include genes, mental disorder or personality; however, environment plays an important role also in contributing to potential drug abuse [25]. Peer pressure and drug availability are primary environmental factors contributing to drug abuse and addiction, especially for adolescents [25]. Adolescents exposed to peer group involved with alcohol and other drugs are more likely to take drugs and drinks. Linked to peer environment, is also the issue of accessibility. The easier it is for a person to obtain drugs, the more likely he will use the drugs. High density neighbourhoods with higher levels of drug availability see a higher level of drug abuse among youngsters [25].

Multiple substance users whose fathers were dead had lower quality of life in the domain of social relation $(\mathrm{p}=$ 0.027). The way fathers communicate with their children is important in the child's emotional and social development. Fathers spend time one-on-one interacting with their children especially the male child. From this interaction the children learn how to regulate their feelings and emotions. When the father dies and there is no surrogate father or guardian they feel empty and hard to adjust. This can trigger emotional problems which results in depression, anxiety and substance abuse. This is the likely cause of lower quality of life in these subjects.

Subjects abusing multiple substances whose fathers had primary school education had low quality of life in social relationship domain $(p=0.006)$. Family socioeconomic status touches many aspect of an adolescent's life. The general idea that socioeconomic status has far-reaching influences can be seen in the sheer variety and number of studies in which it serves as a background factor. Socioeconomic status of family of origin can affect factors from community or neighbourhood characteristics to types of disciplines used [26]. Hill and Duncan found that parents' education, especially father's education, as a measure of socioeconomic status, play an important part in children's educational attainment [27]. There is evidence that majority of lower class students did not show high level of educational achievement, that some just manage to make it [28]. A data from the youth module of the 1997 NHSDA reveals that household socioeconomic status as measured by family income is associated with adolescents' substance use [29]. Being from a lower class family of origin was associated with precocious transitions in all aspects of life including social relationship which was found in this study [30]. Fathers with lower educational achievement are likely to work in low income jobs thus live in a deprived environment. Living in deprivation can directly or indirectly impact on the behaviour such as substance use. These effects are usually felt via mediators such as stress [31]. Stress might push young people towards substance use as a coping mechanism. This will further impair their ability to graduate from school thus attain less educational achievement. This drives them into more substance use which ultimately affects and lowers their physical, psychological, and social relationship. Therefore they drift into lower socioeconomic strata and the cycle continues.

The study found single substance users whose family members were non substance abusers had low quality of life in social relationship domain $(p=0.038)$. Substance abuse has an impact on interpersonal relationship. It can destroy families and lead to disharmony in work place. As an individual abusing single substance with no family member abusing substance, the individual will usually find solace usually in the company of heavy drinkers or drug users. The group will support his/her substance using behaviour and provide the individual with a sense of belonging. By spending much time in his/her new found group of substance abusers, the individual lose their social skills and interpersonal relationship. This will likely be the reason for the finding in this study.

\section{Conclusion}

The study found only social relationship domain on WHOQOL-BREF to be statistically significant in the study. Implication is that family characteristics may be easily ignored and left out of consideration in QOL assessment in substance abusers. Way forward is these are modifiable factors in predicting QOL among substance users which should be assessed.

\section{What Is Already Known}

(1) Substance abuse negatively impact on quality of life

(2) QOL is a major determinant of recovery in substances abuse

(3) Assessing QOL in substance abusers can complement more objective symptom measure which aids recovery

\section{What the Study Adds}

(1) The need for assessment of family characteristics for every substances abuser

(2) Family characteristics as predictors of substance use with its potential impact which are a determinant of recovery.

(3) Family characteristics as predictors of quality of life

\section{Competing Interest}

The authors declare no competing interests. 


\section{Authors' Contribution}

All authors contributed equally in the conception, data collection and drafting of manuscript. All authors reviewed and finalized the manuscript. All authors read and approved the final manuscript.

\section{References}

[1] Elizabeth MT, Alexander BL, Meeyoung OM, HyunSoo K, Suzanne B, Min KJ, et al. Prospective patterns and correlates of quality of life among women in substance abuse treatment. Drug and Alcohol Dependence. 2012; 124(3): 242-249.

[2] The World Health Organization Quality of Life Assessment (WHO QOL): Development and general psychometric properties. Soc Sci Med. 1998; 46(12): 1569-1585.

[3] Laudet AB, White WL. Recovery capital as prospective predictor of sustained recovery, life satisfaction, and stress among former poly-substance users. Subst Use Misuse. 2008; 43(1): $27-54$.

[4] Center for Substance Abuse Treatment. National Summit on Recovery: Conference Report. DHHS Publication No. (SMA) 07-4276. 2007. Rockville, MD: Substance Abuse and Mental Health Services Administration.

[5] Urbanoski KA, Cairney J, Adlaf E, Rush B. Substance abuse and quality of life among severely mentally ill consumers: a longitudinal modelling analysis. Soc Psychiatry Psychiatr Epidemiol. 2007; 42(10): 810-818.

[6] Ingela S, Agneta O. Predictors of Improvement in quality of life of severely mentally ill substance abusers during 18 months of co-operation between Psychiatric and Social services. Soc Psychiatry Psychiatr Epidemiol. 2003; 38(2): 8387.

[7] Stephen P, Ricardo D. Family characteristics and preschool abilities of children of substance abusers. Perceptual and Motor Skills. 1993; 76(3): 912-914.

[8] Irving M, Avraham S. Individual and family characteristics of middle class Adolescents hospitalized for alcohol and other drug abuse. British Journal of Addiction. 1991; 86(11): 14351447.

[9] Carvalho V, Pinsky I, De Souza E Silva R, Carlini-Cotrim B. Drug and alcohol use and family characteristics: a study among Brazilian high- School students. Addiction. 1995; 90(1): 65-72.

[10] Gureje O, Lasebikan VO, Lola K, Makanjuola VA. Life time and 12 month prevalence of mental disorders in Nigerian Survey of Mental Health and Wellbeing. British Journal of Psychiatry. 2006; 188(5): 464-471.

[11] Armiya'u A. Y, Audu MD, Obembe A, Umar UM, Adole O. A study of psychiatric morbidity and comorbid physical illness among convicted and awaiting trial inmates in Jos prison. Journal of Forensic and Legal Medicine. 2013; 20(8): $1048-1051$

[12] WHOQOL Group. WHOQOL Group development of the World Health Organization WHOQOL-BREF quality of life assessment. Psychol Med. 1998; 28(3): 551-558.
[13] American Psychiatric Association. Diagnostic and Statistical Manual of Mental Disorder (DSM IV) $-4^{\text {th }}$ edition. 1994.

[14] Namita PR, Chaturvedi RM. A study of socio-demographic factors contributing to the habit of drug abuse in the urban slum community Mumbai. Biomedical Research. 2010; 21(3): 45-50.

[15] Adelekan M, Makanjuola AB, Ndom RJE, et al. Five yearly monitoring of substance use among secondary school students in Ilorin, Nigeria. West African Journal of Medicine. 2001; 20(1): 28-36.

[16] Amato PR, Sobolewski JM. The effect of divorce and marital discord on adults Children's psychological well-being. American Sociological Review. 2001; 66(6): 900-921.

[17] Jessor R, Jessor S. A. Longitudinal study: Problem behaviour and psychological development. 1997. New York: New York Academic Press Inc.

[18] Chuan-Yu C, Carla LS. Alcohol abuse and health related quality of life among youths in Taiwan. J Adolesc Health. 2006; 39(5): 752

[19] Lamptey JJ. Socio-demographic characteristics of substance abusers admitted at a private specialist clinic. Ghana Med J. 2005; 39(1): 2-7.

[20] Newcomb M. D, Bentler P. M. Substance use and abuse among children and teenagers. AmericanPsychologist. 1989; 44(2): 242-248.

[21] Hawkins JD, Lisher DM, Jenson JM, Catalano RF. Delinquents and drugs: what the evidence suggests about prevention and treatment programming. National Institute on Drug Abuse. (DHHS Publication No. ADM 87-1537). 1987. Washington, DC: U. S. Government Printing Office.

[22] Boys A, Farrell M, Taylor C, Marsden J, Goodman R et al. Psychiatric morbidity and substance use in young people aged 13-15 years: results from the child and adolescent survey on mental health. British Journal of Psychiatry. 2003; 182(6): 509-517.

[23] Igwe WC, Ojinnaka NC. Mental health of adolescents who abuse psychoactive substances in Enugu, Nigeria- A crosssectional study. Italian Journal of Pediatrics. 2010; 36(53): $1-5$.

[24] Laveist TA, Wallace JM. Health risk and inequitable distribution of liquor stores in African American neighbourhood. Social Science and Medicine. 2000; 51(4): 613-617.

[25] Johnson JG, Spitzer RL, William JB, Kroenke K, Linzer M et al. Psychiatry comorbidity, health status, and functional impairment associated with alcohol abuse and dependence in primary care patients: Findings of the PRIME MD-1000 study. J Consult. Clin Psychol. 1995; 63(1): 133-140.

[26] Avenevoli S, Sessa FM, Steinberg L. Family structure, parenting practices, and adolescent adjustment: An ecological examination. In E. M. Hetherington (Ed), Coping with divorce, single parenting, and remarriage. 1999. Mahway, New Jersey: Lawrence Erlbaum Associates, Publishers.

[27] Hill MR, Duncan GJ. Parental family income and the socioeconomic attainment of children. Social Science Research. 1987; 16(1): 39-73. 
[28] Sewell WH, Shah VP. Socioeconomic status, Intelligence, and the Attainment of higher education. Sociology of Education. 1967; 40(1): 1-23.

[29] The National Household Survey on Drug Abuse (NHSDA). 1997 www.samhsa.gov/data/nhsda/Pe1997/popes103.htm.
[30] Candace E, Rob AE, Todd J, Platt S. Indicators of socioeconomic status for adolescents: the WHO Health Behaviour in School-aged Children Survey. Health Educ. Res. 1997; 12(3): 385-397.

[31] Turner RJ, Wheaton B, Lloyd DA. The epidemiology of social stress. American Sociological Review. 1995; 60(1): 104-125. 